\title{
An Algorithm for the Navier-Stokes Problem
}

\author{
F.Z. NOURI and K.AMOURA * \\ * DEPARTEMENT DE MATHEMATIQUES \\ FACULTE DES SCIENCES \\ UNIVERSITE BADJI MOKHTAR \\ BP 12, ANNABA 23000 (ALGERIA).
}

|

RÉSUMÉ. Cette étude est la continuation des travaux [7],[8] et [9] qui sont basés sur l'étude faite par Glowinski \& Al [3] et [4] ainsi que Bernardi \& Al (voir [1] et [2]). Ici nous proposons un Algorithme pour résoudre un problème non-linéaire issu de la mécanique des fluides. Dans [7] nous avons étudié le probléme de Stokes en adaptant la technique de Glowinski, grace à laquelle, on peut découpler la pression de la vitesse lors de la résolution du probléme de Stokes. Dans ce travail, nous étendons notre étude et montrons que cette technique peut être utilisée dans la résolution d'un problème non-linéaire comme les équations de Navier Stokes. Des tests numériques confirment l'intérêt de la discrétisation.

ABSTRACT. This study is a continuation of the one done in [7],[8] and [9] which are based on the work, first derived by Glowinski \& Al in [3] and [4] and also Bernardi \& Al [1] and [2]. Here, we propose an Algorithm to solve a nonlinear problem arising from fluid mechanics. In [7], we have studied Stokes problem by adapting Glowinski technique. This technique is userful as it decouples the pressure from the velocity during the resolution of the Stokes problem. In this paper, we extend our study to show that this technique can be used in solving a nonlinear problem such as the Navier Stokes equations. Numerical experiments confirm the interest of this discretisation.

MOTS-CLÉS : Algorithmes Numériques, Approximations Spectrales, Problèmes de Stokes \& NavierStokes.

KEYWORDS : Numerical Algorithms, Spectral Approximations, Stokes \& Navier Stokes Problems.

|. 


\section{Introduction}

Over the last decade, there has been considerable interest in studying axisymmetric problems and their approximations. These problems have been studied by many authors, namely, Glowinski \& Al [3],[4], Bernardi \& Al [1],[2] and Nouri \& Al [7],[8],[9] and references therein. The numerical discretisations relying on the current, the whirpool and the pressure formulation has first been performed for finite element methods see [10], in the much simpler case of the Stokes problem, and extended to the case of spectral methods [2] and [7], where spectral analogues of Nédelec finite elements [10] are used. Relying on the work in [7] and [8], we propose a discretisation of the Navier Stokes equation in the situation where the domain is axi-symmetric. We present some numerical experiments which confirm the optimality of the discretisation. The analysis of Navier-Stokes equations for incompressible viscous flows in a connected bounded domain can be reduced to analysing the corresponding Stokes problem.

First, we consider the Stokes problem in the tridimensional domain $\widetilde{\Omega}$ that we suppose invariant by rotation around the axis of the cylinder. Then, we use the cylindrical coordinates $(r, \theta, z), r \in I R^{+}, \theta \in[-\pi, \pi]$ and $z \in I R$. The domain $\widetilde{\Omega}$ is denoted by :

$$
\widetilde{\Omega}=\left\{(x, y, z) \in I R^{3} / \quad(r, z) \in \Omega \cup \Gamma_{0} \text { and }-\pi \leq \theta \leq \pi\right\}
$$

where $\Gamma_{0}$ is the interior part of the boundary of $\Omega$ in the axis $\{r=0\}$ and we denote by : $\Gamma=\partial \Omega / \Gamma_{0}$.

First, we introduce the functional spaces :

$$
L_{1}^{2}(\Omega)=\left\{v \text { integrable in } \Omega, \text { such that } \int_{\Omega} v^{2}(r, z) r d r d z<\infty\right\}
$$

with the norm

$$
\|v\|_{L_{1}^{2}(\Omega)}=\left(\int_{\Omega} v^{2}(r, z) r d r d z<\infty\right)^{\frac{1}{2}}, \quad \forall v \in L_{1}^{2}(\Omega) .
$$

and the corresponding weighted Sobolev spaces :

$$
H_{1}^{1}(\Omega)=\left\{v \in L_{1}^{2}(\Omega), \quad \nabla v=\left(\partial_{r} v, \partial_{z} v\right) \in\left(L_{1}^{2}(\Omega)\right)^{2}\right\}
$$


ARIMA - Volume 5-2006

$$
\begin{gathered}
H_{1,0}^{1}(\Omega)=\left\{v \in H_{1}^{1}(\Omega): v=0 \text { on } \partial \Omega / \Gamma_{0}\right\} . \\
V_{1}^{s}(\Omega)=\left\{v \in H_{1}^{s}(\Omega), \quad r^{l+m-s} \partial_{r}^{l} \partial_{z}^{m} v \in L_{1}^{2}(\Omega), 0 \leq l+m \leq s\right\} \\
V_{1,0}^{1}(\Omega)=\left\{v \in V_{1}^{1}(\Omega), \quad v=0 \text { on } \Gamma\right\} .
\end{gathered}
$$

Then, the Stokes problem can be written as :

$$
\left\{\begin{array}{c}
-\nu\left(\partial_{r}^{2} u_{r}+\frac{1}{r} \partial_{r} u_{r}-\frac{1}{r^{2}} u_{r}+\partial_{r}^{2} u_{z}+\partial_{z}^{2} u_{z}\right)+\partial_{r} p=f_{r}, \text { in } \Omega \\
-\nu\left(\partial_{r}^{2} u_{z}+\frac{1}{r} \partial_{r} u_{z}+\partial_{z}^{2} u_{z}\right)+\partial_{z} p=f_{z}, \text { in } \Omega \\
\partial_{r} u_{r}+\frac{1}{r} u_{r}+\partial_{z} u_{z}=0, \text { in } \Omega \\
u_{r}=0, u_{z}=0 \text { on } \Gamma
\end{array}\right.
$$

For the known $f=\left(f_{r}, f_{z}\right) \in\left(V_{0}^{1}\right)^{\prime} \times\left(H_{0}^{1}\right)^{\prime}$, there exists $u=\left(u_{r}, u_{z}\right) \in\left(V_{1,0}^{1}\right) \times$ $\left(H_{1,0}^{1}\right)$. The equation for the divergence can be written as :

$$
\partial_{r}\left(r u_{r}\right)+\partial_{z}\left(r u_{z}\right)=0, \text { in } \Omega
$$

Equation [2] show that the vector $\left(r u_{r}, r u_{z}\right)$ is of divergence free. From theorem 3.1 in chapter 1 of [5], there exists a function $\varphi$ verifying

$$
\left(\begin{array}{c}
r u_{r} \\
r u_{z}
\end{array}\right)=r u=\operatorname{rot}(\varphi)=\left(\begin{array}{c}
\partial_{z} \varphi \\
-\partial_{r} \varphi
\end{array}\right)
$$

By taking $\varphi=r \psi$, we have $\partial_{z} \varphi=r \partial_{z} \psi$ and $-\partial_{r} \varphi=-\psi-r \partial_{r} \psi$, then we set :

$$
\left\{\begin{array}{c}
u_{r}=\partial_{z} \psi \\
u_{z}=-\frac{1}{r}\left(\partial_{r}(r \psi)\right)
\end{array}\right.
$$

Now, let us denote [2] by $\operatorname{div}_{r} u$ which can also be written as

$$
\partial_{r} u_{r}+\frac{1}{r} u_{r}+\partial_{z} u_{z}=0
$$

and by $\operatorname{rot}_{r} \psi$ the vector $\left(\partial_{z} \psi,-\frac{1}{r} \partial_{r}(r \psi)\right)$. We also introduce the operator Rot given by :

$$
\operatorname{Rot}(v)=\partial_{r} v_{z}-\partial_{z} v_{r}, \quad v=\left(v_{r}, v_{z}\right)
$$

\section{ARIMA}


and the operator rot such that for any $\psi$, we have :

$$
r o t \psi=\left(\begin{array}{c}
\partial_{z} \psi \\
-\partial_{r} \psi
\end{array}\right)
$$

We can then write

$$
\operatorname{Rot}(\operatorname{rot} \psi)=-\Delta \psi
$$

\section{Remark 1}

If $\operatorname{div} v=0$, we have $\operatorname{rot} \operatorname{Rot}(v)=-\Delta v$. If we define the operator $\Delta_{r}$ by :

$$
\Delta_{r} \psi=\partial_{r}^{2} \psi+\frac{1}{r} \partial_{r} \psi-\frac{1}{r^{2}} \psi+\partial_{z}^{2} \psi
$$

we can then show that

$$
\operatorname{Rot}\left(\operatorname{rot}_{r} \psi\right)=-\Delta_{r} \psi
$$

(For details see proposition 3.3.2 in [7]).

\section{Continuous Problem}

The question is now what are the necessary boundary conditions to ensure the existence and uniqueness of the current function $\psi$ defined by :

$$
\left\{\begin{array}{c}
u_{r}=\partial_{z} \psi \\
u_{z}=-\frac{1}{r} \partial_{r}(r \psi)
\end{array}\right.
$$

and then deduce its regularity from the regularity of the velocity $u$, the solution of the Stokes problem, see [7] for details. We consider

$$
H_{1}\left(\operatorname{div}_{r}, \Omega\right)=\left\{v \in\left(L_{1}^{2}(\Omega)\right)^{2} / \operatorname{div}_{r} v \in L_{1}^{2}(\Omega)\right\}
$$

with the norm :

$$
\|v\|_{H_{1}\left(d i v_{r}, \Omega\right)}=\left(\|v\|_{\left(L_{1}^{2}(\Omega)\right)^{2}}^{2}+\left\|d i v_{r} v\right\|_{L_{1}^{2}(\Omega)}^{2}\right)^{\frac{1}{2}}
$$

Note that the boundary operator is defined and continuous from $H_{1}^{1}(\Omega)$ onto $H_{1}^{\frac{1}{2}}(\Gamma)$. Note also that $H_{1}^{-\frac{1}{2}}(\Gamma)$ coincides with $H_{1}^{\frac{1}{2}}(\Gamma)$ in the points away from $\bar{\Gamma}_{0} \cap \bar{\Gamma}$. In the same way, we define the normal boundary operator which is continuous from $H_{1}^{1}\left(d i v_{r}, \Omega\right)$ onto $H_{1}^{-\frac{1}{2}}(\Gamma)\left(\right.$ The dual of $\left.H_{1}^{\frac{1}{2}}(\Gamma)\right)$ by : 
ARIMA - Volume 5-2006

$$
\begin{gathered}
\forall v \in H_{1}\left(\operatorname{div}_{r}, \Omega\right) \text { and } q \in H_{1}^{1}(\Omega) \\
(v \cdot n, q)_{\Gamma}=\int_{\Omega}\left(\operatorname{div}_{r} v \cdot q\right)(r, z) r d r d z+\int_{\Omega}(v \cdot \nabla q)(r, z) r d r d z
\end{gathered}
$$

$(., .)_{\Gamma}$ is the scalar product of duality between $H_{1}^{\frac{1}{2}}(\Gamma)$ and $H_{1}^{-\frac{1}{2}}(\Gamma)$ with the measure $d \tau=r d r$ on the side which is parrallel to $o z$ and $=d z$ on the two sides of $\Gamma$.

It has been shown in [7] that :

\section{Proposition 1}

$\forall v \in H_{1}\left(\operatorname{div}_{r}, \Omega\right)$ such that $\operatorname{div} v_{r} v=0$ and $v \cdot n_{\rceil_{\Gamma}}=0, \exists \varphi \in V_{1}^{1}(\Omega)$ such that $v=\operatorname{rot}_{r} \varphi$.

From theorem 3.1 of [5], $\exists \varphi \in H^{1}(\Omega)$ such that $r v=\operatorname{rot} \varphi$. If we set $\Phi=\frac{1}{r} \varphi$ verifying $v=\operatorname{rot}_{r} \Phi$, we have $\partial_{z} \Phi \in L_{1}^{2}(\Omega)$ and $\frac{1}{r} \partial_{r}(r \Phi) \in L_{1}^{2}(\Omega)$. By integrating by parts, we get :

$$
\frac{\partial(r \Phi)}{\partial \tau}=0 \text { on } \Gamma
$$

where $\frac{\partial \varphi}{\partial \tau}$ is the tangential derivative given by :

$$
\frac{\partial \varphi}{\partial \tau}=\nabla \varphi \cdot \tau
$$

The function $r \Phi=C_{i}$ on each connected component $\left.\Gamma_{i}\right\rceil_{i=1}^{I}$ of $\partial \Omega$ where $C_{i}=0$ on all the ones that touch the axis $\{r=0\}$. Hence $\varphi \in V_{1}^{1}(\Omega)$.

Let us now define the boundary conditions for the current function. For, we consider $v \in H_{1}\left(d i v_{r}, \Omega\right)$ such that $d i v_{r} v=0$. Let us denote by $\varphi$ the function associated to $v$, From proposition 1 , we have $r \varphi=c$ (a constant) on $\Gamma$. However $\Gamma$ touch the axis $\{r=0\}$, hence $c=0$ and $\left.\varphi\right|_{\Gamma}=0$. We set the following result :

\section{Corollary 2}

Let $v \in H_{1}\left(\operatorname{div}_{r}, \Omega\right)$ such that $\operatorname{div}_{r} v=0$ and $\left.v \cdot n\right|_{\Gamma}=0$. If $\varphi$ is the current function associated to $v$, then $\varphi$ is the solution of the following problem :

$$
\left\{\begin{array}{c}
\varphi \in V_{1,0}^{1}(\Omega) \\
\int\left(\operatorname{rot}_{r} \varphi \cdot \operatorname{rot}_{r} \chi\right)(r, z) r d r d z=\int\left(v \cdot \operatorname{rot}_{r} \chi\right)(r, z) r d r d z, \forall \chi \in V_{1,0}^{1}(\Omega)
\end{array}\right.
$$

To study this problem (see [5] and [7]), we introduced the space : 


$$
H_{1}(\operatorname{rot}, \Omega)=\left\{v \in\left(L_{1}^{2}(\Omega)\right)^{2} / \operatorname{rot}(v) \in L_{1}^{2}(\Omega)\right\}
$$

and we define the boundary operator from $H_{1}(r o t, \Omega)$ onto $H^{-\frac{1}{2}}(\Gamma)$ as :

$$
\begin{gathered}
\forall v \in H_{1}(\operatorname{rot}, \Omega), \forall \chi \in V_{1}^{1}(\Omega), \\
\prec v \cdot \tau, \chi \succ_{\Gamma}=\int_{\Omega}\left(\operatorname{verot}_{r} \chi\right)(r, z) r d r d z-\int_{\Omega} \operatorname{rot}(v) \chi(r, z) r d r d z
\end{gathered}
$$

Hence we can write :

$$
\left\{\begin{array}{c}
-\Delta_{r} \varphi=\operatorname{rot}(v) \text { in } D^{\prime}(\Omega) \\
\left.\varphi\right|_{\Gamma}=0 \\
\left.\left(\frac{1}{r} \frac{\partial(r \varphi)}{\partial n}+v \cdot \tau\right)\right|_{\Gamma}=0
\end{array}\right.
$$

where $\frac{\partial \varphi}{\partial n}=\nabla \varphi \cdot n$.

\section{Proposition 3}

We suppose that $v \in V_{1,0}^{1}(\Omega) \times H_{1,0}^{1}(\Omega) / \operatorname{div}_{r}(v)=0$ and $\left.v \cdot n\right|_{\Gamma}=0$, then there exists a current function $\varphi$ in $V_{1}^{2}(\Omega) / v=\operatorname{rot} \varphi$ verifying $\varphi=0$ on $\partial \Omega$ and $\frac{\partial \varphi}{\partial n}=0$ on $\Gamma$, where $V_{1}^{2}(\Omega)$ is defined by :

$$
V_{1}^{2}(\Omega)=\left\{\varphi \in L_{1}^{2}(\Omega) / r^{l+m-2} \partial_{r}^{l} \partial_{z}^{m} \varphi \in L_{1}^{2}(\Omega), \quad 0 \preceq(l+m) \preceq 2\right\} .
$$

\section{Remark 2}

For the proof of this proposition, we use Hardy inequality (See [7] and references therein). Remark 3

In [8], we showed how we can generalise this result.

\section{Current-Whirpool function formulation}

In this section, we introduce a new unknown called whirpool function $\omega=\operatorname{rot}(u)$ which gives :

$$
-\Delta u=\operatorname{rot}_{r} \omega
$$


ARIMA - Volume 5 - 2006

$\Delta$ is applied to $\left(u_{r}, u_{z}\right)$ in the two first equations of [1].

If we substitute this result in [1], we obtain

$$
\left\{\begin{array}{c}
-v \partial_{z} \omega+\partial_{r} p=f_{r} \\
-v \frac{1}{r} \partial_{r}(r \omega)+\partial_{z} p=f_{z}
\end{array}\right.
$$

The interesting point of this formulation is to decouple the pressure from the velocity to obtain a separate problem for the pressure. We have

$$
-\Delta_{r} \omega=\frac{1}{v}\left(\partial_{z} f_{r}-\partial_{r} f_{z}\right)
$$

where $u=\operatorname{rot}_{r} \psi$ and $\omega=-\Delta_{r} \psi$.

Hence we can conclude that this formulation is equivalent to the one derived by Glowinski and Pironneau in [3] and [4].

\subsection{The reduced Problem}

The advantage of this decoupling is to write [1] as :

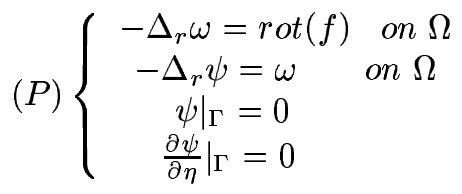

Problem $(\mathrm{P})$ is exactly a Dirichlet problem for the biharmonic operator $\Delta_{r}^{2}$, where the solution is the current function $\psi$ such that :

$$
\left.P_{0}\right)\left\{\begin{array}{c}
-\Delta_{r}^{2} \psi=\operatorname{rot}(f) \text { on } \Omega \\
\left.\psi\right|_{\Gamma}=0 \\
\frac{\partial \psi}{\partial n} \mid \Gamma=0
\end{array}\right.
$$

Problem $\left(P_{0}\right)$ can be written in a matrix form as :

$$
D U=F
$$

where $D$ is the matrix with the entries $a_{N}\left(h_{i} l_{j}, h_{r} l_{s}\right)$ defined by :

$$
a_{N}\left(h_{i} l_{j}, h_{r} l_{s}\right)=\left(h_{i}^{\prime} l_{j}, h_{r}^{\prime} l_{s}\right)_{N}+\left(h_{i} l_{j}^{\prime}, h_{r} l_{s}^{\prime}\right)_{N}+\left(\frac{1}{r} h_{i} l_{j}, \frac{1}{r} h_{r} l_{s}\right)_{N}
$$


where $(u, v)_{N}$ is given by :

$$
(u, v)_{N}=\sum_{i=1}^{N+1} \sum_{j=0}^{N} u\left(\zeta_{i}, \xi_{j}\right) v\left(\zeta_{i}, \xi_{j}\right) \omega_{i} \rho_{j}, 2 \leq i, r \leq N, 1 \leq j, s \leq N-1 .
$$

Here $h_{i}$ respectively $l_{j}$, are the Lagrange polynomials associated at the nodes $\zeta_{i}$ and $\xi_{j}, 1 \leq i \leq N+1$ and $0 \leq j \leq N$.

$U$ is constructed by the values of the solution $u_{N}$ at the nodes $\left(\zeta_{i}, \xi_{j}\right)$ and $F$ by the terms, $f\left(\zeta_{i}, \xi_{j}\right) \omega_{i} \rho_{j}$, where $\omega_{i}$ and $\rho_{j}$ are the associated weights.

We remark that $D$ is a symmetric positive definite matrix, therefore the gradient conjugate Algorithm can be used. A study of the continuous problem was done by [2] and [3], and the spectral discretisation was studied in [7] and [9]. Here we would like to extend this study to the Navier-Stokes equation.

\section{Numerical Study}

In the same way we have applied the above analysis to a nonlinear problem (see [9] for details) and analogues results have been found. Using the above formulation, we can write the Navier Stokes equation as :

$$
(P)\left\{\begin{array}{rrr}
-\nu \Delta_{r} \omega+\nabla_{r} \psi \cdot \operatorname{rot}_{r} \omega=\operatorname{rot}(f) \text { on } \Omega \\
-\Delta_{r} \psi=\omega & \text { on } \Omega \\
\left.\psi\right|_{\Gamma}=0 & \\
\left.\frac{\partial \psi}{\partial \eta}\right|_{\Gamma}=0 &
\end{array}\right.
$$

where

$$
\nabla_{r} \varphi=\left(\frac{1}{r} \partial_{r}(r \varphi), \partial_{z} \varphi\right)
$$

and

$$
\operatorname{rot}_{r} \varphi=\left(\partial_{z} \varphi,-\frac{1}{r} \partial_{r}(r \varphi)\right) \text { for any regular function } \varphi .
$$

Therefore we set an Algorithm to solve problem (P).

\subsection{Algorithm}

We propose the following Algorithm

- Approximate $\psi$ and $\left\{\psi_{m}\right\}_{m=1}^{M}$ by :

$$
\psi_{m+1}=\psi_{m}+\alpha\left(\bar{\psi}_{m}-\psi_{m}\right) .
$$

- Approximate $\omega$ and $\left\{\omega_{m}\right\}_{m=1}^{M}$ by : 
ARIMA - Volume 5 - 2006

$$
\omega_{m+1}=\omega_{m}+\alpha\left(\bar{\omega}_{m}-\omega_{m}\right) .
$$

$\omega_{m}$ and $\psi_{m}$ are solutions of

$$
\left\{\begin{array}{c}
-\nu \Delta_{r} \bar{\omega}_{m}=-\frac{1}{r} \partial_{r}\left(r \psi_{m}\right) \partial_{z} \omega_{m}-\partial_{r}\left(r \omega_{m}\right) \partial_{z} \psi_{m}+\operatorname{rot}(f) \\
-\Delta_{r} \bar{\psi}_{m}=\bar{\omega}_{m} \\
\left.\bar{\psi}_{m}\right|_{\Gamma}=0 \quad \text { and }\left.\quad \frac{\partial \bar{\omega}_{m}}{\partial \eta}\right|_{\Gamma}=0
\end{array}\right.
$$

Hence the nonlinear problem can be solved as four problems for $\Delta_{r}$.

For the numerical tests, we have taken the following examples.

\section{Example 1}

The first test is the function

$$
\Psi=\cos (\pi r) \sin (\pi z)\left(1-r^{2}\right)^{3}\left(1-z^{2}\right)
$$

\section{Example 2}

The second test is a singular stream

$$
\Psi=(1-r)^{3}(1+r)^{11 / 2}\left(1-z^{2}\right)
$$

Numerical tests were carried out in the IRISA laboratory in Rennes (France)

\subsection{Numerical Results}

Numerical experiments turn out to be in agreement with the analysis done in [7] and [9]. As shown in Figures 1, 2 and 3, numerical results for examples 1 and 2 were compared to the exact solutions. The error plots are in the $L^{2}$-norm. We noticed that :

1 - The error analysis for the current function is qualitatively the same for both examples 1 and 2 (Figure 3 ).

2- The choice of the basis does not affect the quality of our numerical results. However, when using the nodes to be the roots or the extremums of Chebyshev polynomials, better results were found.

3- From Figure 2, where numerical investigations for a singular problem (example 2) was considered, the error has a spectral behavior, that is to say, we do not have any spurious modes that destruct the solution.

4- Many choices for the relaxation parameter were used and the optimal value was $\alpha=$ 0.5 . For the choice of parameters for our plots, we have taken the viscosity $\nu=1$, the 


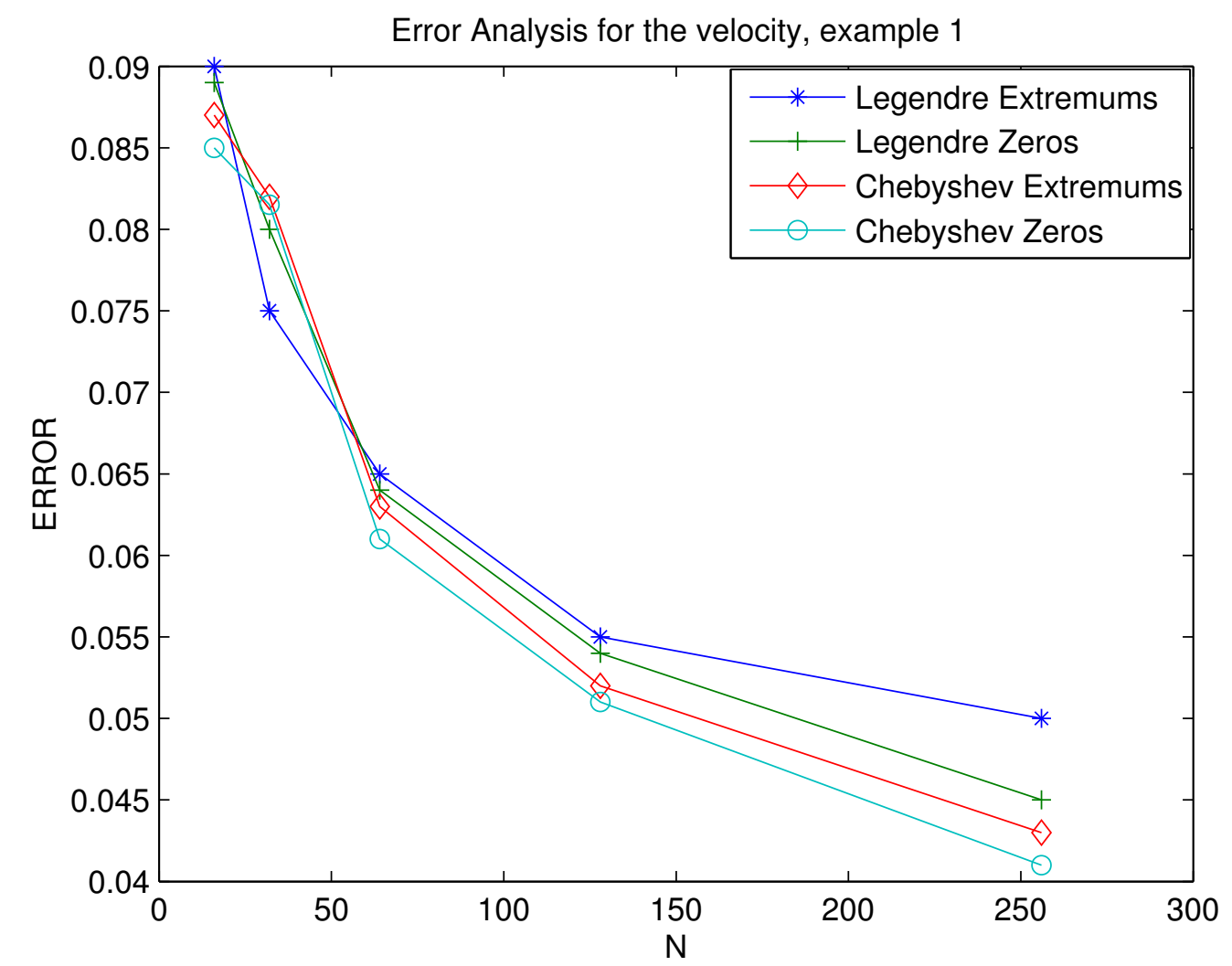

Figure 1. Error Analysis for the velovity, Example 1

relaxation parameter $\alpha=0.5$.

A Study of the pressure is under consideration.

\section{Conclusion}

In this paper, we proposed a new technique similar to the one by Glowinski \& Al [5] and show that we can reduce Stokes and Navier-Stokes problems to a set of saddle point ones; where the unknowns are the current and whirpool functions. Here the pressure is completely decoupled from the velocity to give a simple Dirichlet problem to be solved. 

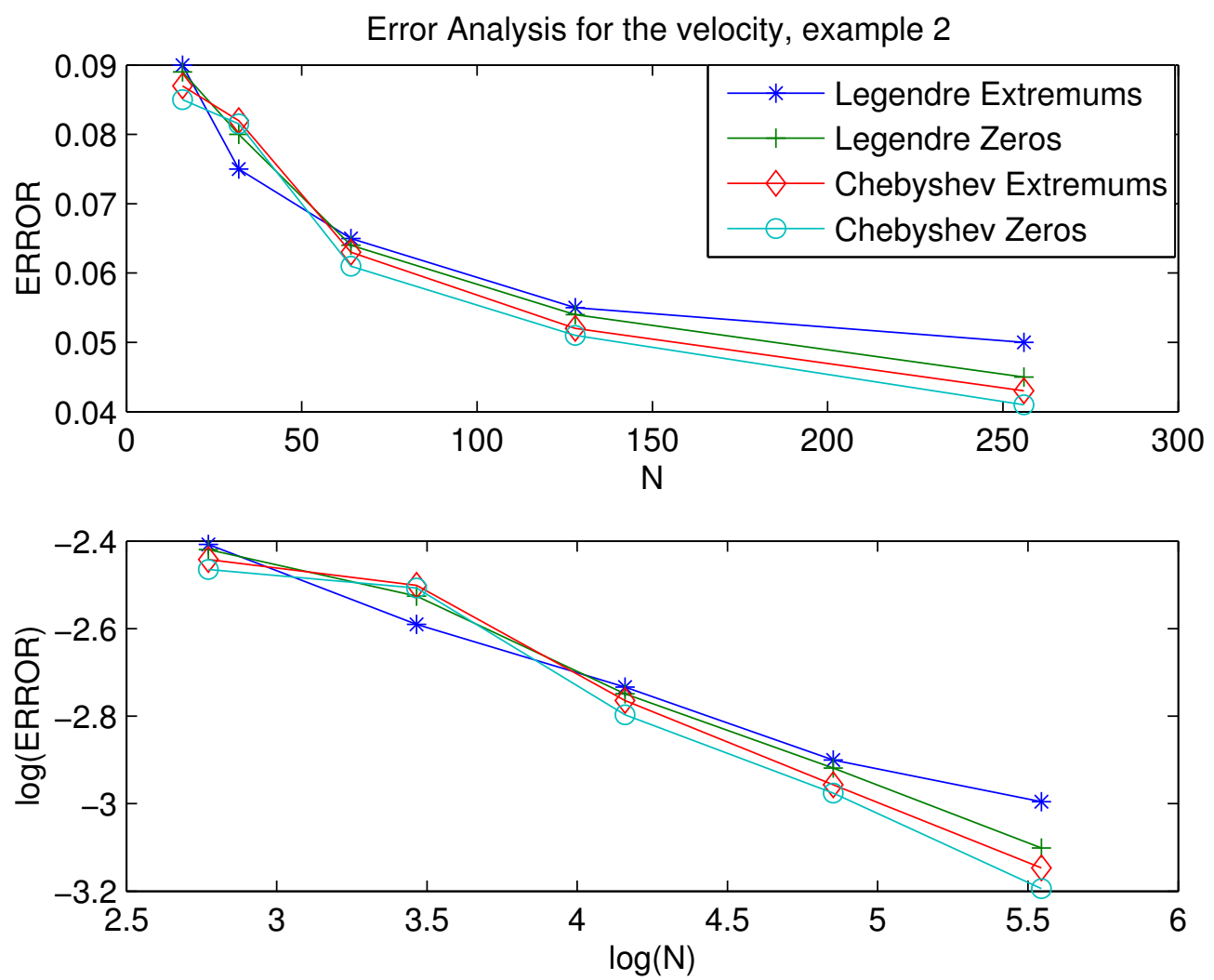

Figure 2. Error Analysis for the velocity, Example 2

It is clear that this technique can be very useful in nonlinear cases as it has been shown above for the Navier Stokes equations. Optimal results were found.

Aknowelegement : We are very grateful for Professor Bernard Philippe for providing the numerical facilities at the IRISA.The authors are also grateful to professor Bernardi for the fruitful discussions during her visit in our department in September 2005. We also wish to thank the referees for their comments and suggestions. 


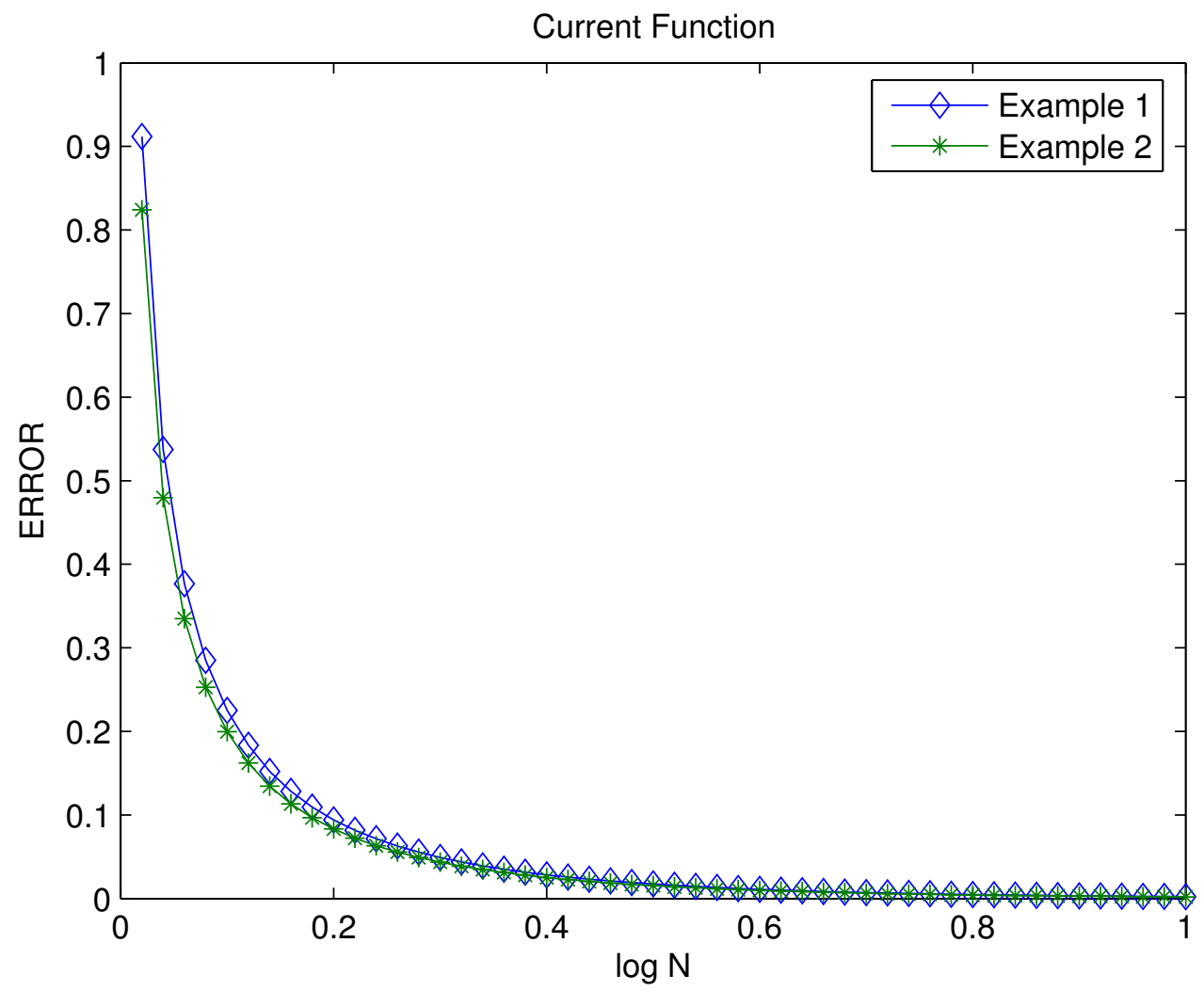

Figure 3. Chebyshev Spectral Error Analysis for the current function

\section{Bibliographie}

[1] C. Bernardi and Y. Maday, Spectral Methods, Handbook of Numerical Analysis, Edited by P.G. Ciarlet and J.L. Lions, 1997 Elsevier Science ;

[2] C. Bernardi and M. Dauge and Y. Maday, Spectral Methods for axi-symmetric Problems, Numerical algorithms and tests due to Majdi Azaiz, 1999, Edition Elsevier Sciences Medicales.

[3]R.Glowinski, Numerical Methods for nonlinear problems, Springer Verlag (1982).

[4] R. Glowinski \& O.Pironneau, Numerical Methods for the First Biharmonic equation, SIAM SIREV, Vol 21 No 2 (1979).

[5] V. Girault, P.A. Raviart, Finite Element Methods for Navier-Stokes Equations, Theory and Algorithms, Springer Verlag, New-York (1986). 
ARIMA - Volume 5 - 2006

[6] F.Z. Nouri,New Fitting for a Spectral Method for the solution of Stokes problem Presented in the Bienal Conference of Numerical Analysis, Abstracts, Dundee NA099.

[7] F.Z. Nouri \& A.Boutaghou Stokes problem in the case of axi-symmetric Data, Far East Int. J. Of Applied mathematics, vol21, No2, 2005.

[8] F.Z. Nouri and K. Amoura,Glowinski Technique for the Stokes problem, Accepted in Revue des Sciences et Technologie, Constantine University (Algeria), to appear.

[9] F.Z. Nouri \& A. Boutagou, Finite Spectral Method for axi-symmetric Elliptic Problems, Presented in the Bienal Conference of Numerical Analysis,Dundee 2001.to appear in S.A.S. Int. Publications, Journal of Analysis and Applications.

[10]S. Salmon, Développement numérique de la formulation tourbillon-vitesse-pression pour le problème de Stokes, Thèse Université Pierre et Marie Curie, Paris 1999. 